\title{
CIRURGIA DE CONTROLE DE DANOS: EMBASAMENTO TEÓRICO E IMPLICAÇÕES PRÁTICAS
}

\section{ARTIGO DE REVISÃO}

PEREIRA, Fernanda Cândido ${ }^{1}$

SILVA, Ana Beatriz Vieira Da ${ }^{2}$

SOUZA, Bruno Pithon Costa ${ }^{3}$

COSTA, Felipe Martins ${ }^{4}$

CORDEIRO, lago Alvares ${ }^{5}$

FERREIRA, Karoline Fraga ${ }^{6}$

LIMA, Letícia Alves de ${ }^{7}$

MALHEIROS, Matheus José Barroso ${ }^{8}$

${ }^{1}$ Discente do curso de Medicina do Centro Universitário UNINTA, Sobral - CE.

2 Discente do curso de Medicina da Universidade Federal do Amazonas.

${ }^{3}$ Discente do curso de Medicina da Faculdade de Ciências Médicas e da Saúde de Juiz de Fora - Suprema, Juiz de Fora - MG.

${ }^{4}$ Discente do curso de Medicina da Universidade Estadual de Santa Cruz, Ilhéus BA.

${ }^{5}$ Discente do curso de Medicina da Faculdade de Ciências Médicas e da Saúde de Juiz de Fora - Suprema, Juiz de Fora - MG.

${ }^{6}$ Discente do curso de Medicina do Centro Universitário UNIFAMINAS, Muriae - MG.

7 Discente do curso de Medicina do Centro Universitário UNINTA, Sobral - CE.

${ }^{8}$ Discente do curso de Medicina da Universidade Federal do Amazonas (Manaus). 
PEREIRA, Thiago Toledo Martins ${ }^{9}$

MOREIRA, Geterson Bezerra ${ }^{10}$

PEREIRA, Fernanda Cândido. Et al. Cirurgia de controle de danos: Embasamento teórico e implicações práticas. Revista Científica Multidisciplinar Núcleo do Conhecimento. Ano 06, Ed. 01, Vol. 06, pp. 53-73. Janeiro de 2021. ISSN: 2448-0959, Link de acesso: https://www.nucleodoconhecimento.com.br/saude/implicacoespraticas

\section{RESUMO}

Introdução: A cirurgia de controle de danos é uma modalidade cirúrgica que abrevia o tempo de operação e renuncia o reparo imediato de todas as injúrias, com o objetivo de reestabelecer os parâmetros fisiológicos no paciente instável hemodinamicamente. Indivíduos que apresentam a acidose metabólica, hipotermia e coagulopatia, a denominada tríade mortal do trauma, são os principais candidatos à essa abordagem. Diante da alta incidência de traumas e patologias que exigem o controle imediato de lesões potencialmente fatais e da escassez de estudos acerca do tema, esta revisão objetiva realizar um levantamento de dados da literatura acerca do controle de danos cirúrgico, que tem se mostrado de extrema importância e impacto na redução da mortalidade dos pacientes graves. Métodos: A busca dos artigos foi realizada nas plataformas Pubmed e BVS, utilizando os descritores "cirurgia" e "controle de danos", contidos no DeCS (Descritores em Ciências da Saúde) e correspondentes à questão de pesquisa. Foram encontrados 63 estudos ao total. A aplicação de critérios de inserção e exclusão, leitura e seleção dos estudos,

${ }^{9}$ Discente do curso de Medicina da Faculdade de ciências médicas e da saúde de Juiz de Fora - Suprema, Juiz de Fora - MG.

${ }^{10}$ Orientador. Docente do curso de Medicina do Centro Universitário UNINTA, Sobral - CE. Cirurgião geral pela Santa Casa de Misericórdia de Sobral- Ceará. Coordenador da residência de Cirurgia da Santa Casa de Misericórdia de Sobral- UFC, Sobral Ceará. 
resultaram, por fim, na inclusão de 9 artigos na presente revisão. Resultados: Dentre os artigos analisados, 22,2\% dos estudos investigaram as indicações e a importância da decisão por controlar os danos cirurgicamente. O impacto dessa abordagem na redução da mortalidade dos doentes traumatizados foi explanado em 33,3\% dos estudos incluídos. Os estudos relataram sobrevida maior do que $80 \%$ dos pacientes submetidos à essa modalidade terapêutica. $O$ mecanismo de trauma mais comum associado à necessidade de controle de danos foi 0 traumatismo penetrante, como os ferimentos por arma de fogo. Quanto aos motivos que levaram à indicação dessa abordagem, os estudos relataram instabilidade hemodinâmica como sendo o principal deles, além da magnitude das lesões. Conclusões: Diante dos dados coletados da literatura e explanados neste estudo, salienta-se a importância de os cirurgiões conhecerem as implicações teóricas e práticas da cirurgia de controle de danos, a fim de reconhecer prontamente o paciente que necessita dessa abordagem, indicá-la em tempo oportuno e realizá-la efetivamente, elevando a sobrevida dos pacientes graves.

Palavras-Chave: Cirurgia, controle de danos, tríade letal, hemorragia, choque.

\section{INTRODUÇÃO}

A cirurgia de controle de danos é uma modalidade cirúrgica que abrevia o tempo de operação e renuncia o reparo imediato de todas as injúrias, com o objetivo de restabelecer os parâmetros fisiológicos no paciente instável hemodinamicamente (PIMENTEL et al., 2018). Essa estratégia foi utilizada inicialmente para abordar pacientes vítimas de trauma, principalmente abdominal, todavia, atualmente é utilizada em lesões ortopédicas, torácicas e vasculares (EDELMUTH; BUSCARIOLLI; RIBEIRO JUNIOR, 2013).

A cirurgia de controle de danos foi cunhada em 1993, com o artigo de referência de Rotondo et al. (1993) mostrando uma melhora de sete vezes (11\% a $77 \%$ ) na sobrevida de pacientes com lesão visceral e vascular combinada submetidos à essa abordagem (ROTONDO et al., 1993). Essa redução da mortalidade pode ser 
compreendida a partir do entendimento das diferenças entre o paciente de cirurgia eletiva e o paciente vítima de trauma, tanto para a fisiologia quanto para a anatomia. O primeiro geralmente tem um único distúrbio, com ausência de hemorragia descontrolada ou contaminação durante o procedimento. O paciente vítima de trauma, por sua vez, pode sofrer de lesões em diferentes topografias, com perda de fisiologia devido ao sangramento contínuo e contaminação.

Dessa forma, a fisiologia do paciente é o indicador fundamental para avaliar a necessidade da cirurgia de controle de danos (JÚNIOR, 2014). Indivíduos que apresentam a tríade letal do trauma (acidose metabólica, hipotermia e coagulopatia) são os principais candidatos à essa abordagem. Pacientes que apresentam lesões com penetração múltipla, contusas de alta energia, também realizam esse tipo de estratégia cirúrgica preferencialmente (WAIBEL; ROTONDO, 2012). Ademais, essa modalidade terapêutica é cada vez mais utilizada em situações não traumáticas em pacientes com indicadores fisiológicos muito alterados (EDELMUTH; BUSCARIOLLI; RIBEIRO JUNIOR, 2013). Nesse ínterim, pacientes com patologias como abdome agudo por úlcera péptica ou divertículo intestinal perfurados, lesões iatrogênicas como as lacerações de alças intestinais e ligadura de ureter em cirurgias pélvicas, roturas vasculares associadas a distúrbios do tecido conjuntivo, entre outras situações clínicocirúrgicas, também podem se beneficiar de abordagem abreviada para controle de danos.

A estratégia para o controle efetivo dos danos possui 5 etapas cruciais: o estágio 1 , a indicação, no qual deve-se avaliar a condição clínica e os parâmetros do paciente para realizar o procedimento, o estágio 2 (laparotomia abreviada), no qual realiza-se o controle das principais causas potenciais de óbito no trauma: o sangramento e a contaminação, o estágio 3 , no qual são realizadas as medidas de clínicas de ressuscitação para normalização dos parâmetros, como tipo de anestesia apropriada, que possibilite a hipotensão permissiva, o aquecimento do doente, e a transferência à Unidade de Terapia Intensiva (UTI), o estágio 4, que diz respeito às reabordagens cirúrgicas para o tratamento definitivo das lesões, e o estágio 5 ou reabilitação, com 
as terapias psicológica, fisioterápica, fonoaudiológica, entre outras. (JÚNIOR et al., 2014).

Os procedimentos realizados visam, de forma prioritária, o controle dos sangramentos, infecções, contaminação cavitária e derramamentos de conteúdo gastrointestinal, biliar ou vesical na cavidade peritoneal, fatores que causam irritação e peritonite (GERMANOS et al., 2008). Um exemplo de técnica de controle de dano é a laparotomia que tem como definição o controle da hemorragia, prevenção da contaminação peritoneal e proteção contra novos agravos. Muitas vezes são necessárias reabordagens cirúrgicas, para reconstruir o trânsito intestinal, restaurar a continuidade de vasos sanguíneos, reavaliar a viabilidade de vísceras ou para controle da contaminação. As técnicas para fechamento temporário da parede abdominal sem tensão, facilitam o acesso à cavidade peritoneal nos subsequentes tempos cirúrgicos (SIMÃO et al., 2013).

A estratégia para essa cirurgia está relacionada com uma elevada taxa de mortalidade, de 35\%, provavelmente pela gravidade que os pacientes a ela são submetidos (PIMENTEL et al., 2018). Pressão arterial sistólica em índices decrescentes relacionados a elevados níveis de lactato sérico, acidose metabólica grave, tempo de atividade da protombina (TAP) alargado, são parâmetros associados a uma taxa de mortalidade aumentada (PIMENTEL et al., 2018). Assim, a cirurgia de controle de danos busca intervir em tempo oportuno, de modo a evitar a progressão do quadro clínico do paciente para a tríade mortal que pode, rapidamente, levá-lo à óbito, sendo essa a função primordial desse tipo de abordagem, que, quando bem indicada e realizada, salva a vida do paciente em risco iminente de morte.

Diante da alta incidência de traumas e patologias que exigem o controle imediato de lesões potencialmente fatais, é fundamental o conhecimento sobre a cirurgia de controle de danos, tema no qual não há muitos trabalhos publicados. Nesse contexto, este estudo objetiva realizar um levantamento de informações a respeito dessa modalidade cirúrgica, que tem se mostrado de extrema importância e impacto na redução da mortalidade dos pacientes graves. 


\section{METODOLOGIA}

Trata-se de uma revisão da literatura do tipo integrativa, de abordagem qualitativa e natureza aplicada, que objetiva a explanação e análise das informações coletadas acerca da cirurgia de controle de danos.

A busca dos artigos foi realizada utilizando os descritores "cirurgia" e "controle de danos", contidos no DeCS (Descritores em Ciências da Saúde) e correspondentes à questão de pesquisa, empregados em três idiomas: português, inglês e espanhol. Esses descritores junto ao operador booleano "AND" compuseram a fórmula de busca, para utilização nas bases de dados PubMed e BVS.

A pesquisas realizadas nas plataformas de dados resultaram em um total de 4 artigos no PubMed e 59 artigos no BVS. A leitura do título e resumo permitiu a seleção de estudos que abordavam o tema e que condiziam ao objetivo da revisão. Foram empregados como critérios de inclusão os estudos que correspondiam à temática e à finalidade da pesquisa, os trabalhos escritos em português, inglês e espanhol, estudos em humanos e indexados nas plataformas de busca nos últimos 10 anos (2010 a 2020), estando disponíveis para leitura na íntegra. Foram excluídos os artigos que não respondiam nem abordavam a questão de pesquisa e os trabalhos não disponíveis para a leitura na íntegra.

Salientamos que o presente estudo não apresenta caráter prático, não sendo, portanto, necessária a submissão ao Comitê de Ética em Pesquisa (CEP).

Para a explanação dos achados desta pesquisa, os resultados foram esquematizados em tabela, na qual foram inseridos os principais dados de cada um dos artigos incluídos na revisão, como o título, a autoria, ano de publicação, país e os principais resultados encontrados. 


\section{RESULTADOS}

Tabela I - Cirurgia de controle de danos: embasamento teórico e implicações práticas.

\begin{tabular}{|c|c|c|c|}
\hline & Título & $\begin{array}{l}\text { Autores, ano } \\
\text { de } \\
\text { publicação e } \\
\text { país }\end{array}$ & Principais resultados \\
\hline $\begin{array}{l}\text { ARTIG } \\
01\end{array}$ & $\begin{array}{l}\text { Estudo } \\
\text { comparativo de } \\
\text { técnicas de } \\
\text { fechamento } \\
\text { temporário da } \\
\text { cavidade } \\
\text { abdominal para } \\
\text { controle de } \\
\text { danos }\end{array}$ & $\begin{array}{l}\text { RIBEIRO JR } \\
\text { et al., 2016, } \\
\text { Brasil }\end{array}$ & $\begin{array}{l}\text { A técnica VAC foi superior em relação } \\
\text { às técnicas de bolsa Bogotá e de } \\
\text { Barker, por apresentar benefícios } \\
\text { como menor ocorrência } \\
\text { de fístulas, infecções e outras } \\
\text { complicações, menor morbimortalida } \\
\text { de, melhor } \\
\text { controle do líquido do terceiro espaço } \\
\text { e maior facilidade } \\
\text { no fechamento primário da parede } \\
\text { abdominal e deve ser, sempre que } \\
\text { possível, a terapia de escolha nos } \\
\text { casos em que se opta pela } \\
\text { manutenção da laparostomia. }\end{array}$ \\
\hline $\begin{array}{l}\text { ARTIG } \\
02\end{array}$ & $\begin{array}{l}\text { Cirurgia de } \\
\text { controle de } \\
\text { danos torácicos }\end{array}$ & $\begin{array}{l}\text { GONCALVES; } \\
\text { SAAD JR, } \\
\text { 2016, Brasil }\end{array}$ & $\begin{array}{l}\text { Se tratando das lesões torácicas, } \\
\text { manobras relativamente simples } \\
\text { podem diminuir o tempo cirúrgico de } \\
\text { pacientes graves. Toracotomia de } \\
\text { emergência possui como objetivos } \\
\text { primordiais o alívio do tamponamento } \\
\text { cardíaco, controle da ferida cardíaca } \\
\text { ou exsanguinante devido a lesão } \\
\text { vascular, pinçamento da aorta } \\
\text { descendente para aumentar o fluxo }\end{array}$ \\
\hline
\end{tabular}




\begin{tabular}{|c|c|c|c|}
\hline & & & $\begin{array}{l}\text { sanguíneo coronário e cerebral, } \\
\text { compressões cardíacas internas e } \\
\text { oclusão do hilo pulmonar para diminuir } \\
\text { o risco de embolia gasosa e de } \\
\text { sangramento, no caso de lesões } \\
\text { pulmonares graves. }\end{array}$ \\
\hline $\begin{array}{l}\text { ARTIG } \\
03\end{array}$ & $\begin{array}{l}\text { Cirurgia para } \\
\text { controle de } \\
\text { danos: estado } \\
\text { atual }\end{array}$ & $\begin{array}{l}\text { EDELMUTH; } \\
\text { BUSCARIOLL } \\
\text { I; RIBEIRO } \\
\text { JUNIOR, } \\
\text { 2013, Brasil }\end{array}$ & $\begin{array}{l}\text { A experiência clínico-cirúrgica, até } \\
\text { hoje, demonstra que quando a cirurgia } \\
\text { feita para controle de danos é } \\
\text { realizada de forma correta e, mais } \\
\text { importante, quando bem indicada, } \\
\text { reduz a mortalidade em pacientes } \\
\text { gravemente feridos. }\end{array}$ \\
\hline $\begin{array}{l}\text { ARTIG } \\
04\end{array}$ & $\begin{array}{l}\text { Cirurgia de } \\
\text { controle de } \\
\text { danos: estamos } \\
\text { perdendo o } \\
\text { controle sobre } \\
\text { as indicações? }\end{array}$ & $\begin{array}{l}\text { PIMENTEL et } \\
\text { al., 2018, } \\
\text { Brasil }\end{array}$ & $\begin{array}{l}\text { Na maioria dos casos, houve } \\
\text { indicação precoce para cirurgia de } \\
\text { controle de danos, baseada } \\
\text { principalmente no estado } \\
\text { hemodinâmico e na gravidade das } \\
\text { lesões, e em } 65,2 \% \text {, a decisão foi } \\
\text { compatível com alterações em dados } \\
\text { hemodinâmicos e laboratoriais } \\
\text { objetivos. }\end{array}$ \\
\hline $\begin{array}{l}\text { ARTIG } \\
\text { O } 5\end{array}$ & $\begin{array}{l}\text { Avaliação } \\
\text { epidemiológica } \\
\text { de vítimas de } \\
\text { trauma } \\
\text { hepático em } \\
\text { cirurgia }\end{array}$ & $\begin{array}{l}\text { KALIL; } \\
\text { AMARAL, } \\
\text { 2016, Brasil }\end{array}$ & $\begin{array}{l}\text { A cirurgia de controle de danos foi } \\
\text { realizada em sete casos }(6,54 \%) \text { e a } \\
\text { sobrevida dos pacientes foi de } 100 \% \text {, } \\
\text { o que confirma o controle de danos } \\
\text { como medida que eleva a sobrevida } \\
\text { de pacientes gravemente feridos e } \\
\text { que enfrentam a chamada tríade da } \\
\text { morte - acidose metabólica, } \\
\text { hipotermia e coagulopatia. }\end{array}$ \\
\hline
\end{tabular}




\begin{tabular}{|c|c|c|c|}
\hline $\begin{array}{l}\text { ARTIG } \\
06\end{array}$ & $\begin{array}{l}\text { Controle de } \\
\text { danos em } \\
\text { fraturas } \\
\text { instáveis } \\
\text { torácicas e } \\
\text { lombares no } \\
\text { politraumatism } \\
\text { o. Revisão } \\
\text { sistemática }\end{array}$ & $\begin{array}{l}\text { CHAVEZ et } \\
\text { al., 2015, } \\
\text { México }\end{array}$ & $\begin{array}{l}\text { A estabilização da fratura, no que } \\
\text { concerne ao controle de danos, nas } \\
\text { primeiras } 72 \text { horas da lesão, está } \\
\text { associada à menor incidência de } \\
\text { complicações, permanência } \\
\text { hospitalar, permanência na unidade } \\
\text { de cuidados intensivos e à menor } \\
\text { mortalidade. }\end{array}$ \\
\hline $\begin{array}{l}\text { ARTIG } \\
07\end{array}$ & $\begin{array}{l}\text { Controle de } \\
\text { danos: uma luz } \\
\text { no fim do túnel }\end{array}$ & $\begin{array}{l}\text { JÚNIOR, } \\
\text { 2014, Brasil }\end{array}$ & $\begin{array}{l}\text { A laparotomia para controle de danos } \\
\text { tem proporcionado índice de } \\
\text { sobrevida melhor do que o esperado } \\
\text { para o trauma abdominal e sua } \\
\text { aplicabilidade tem sido estendida para } \\
\text { englobar o trauma torácico e } \\
\text { ortopédico. }\end{array}$ \\
\hline $\begin{array}{l}\text { ARTIG } \\
08\end{array}$ & $\begin{array}{l}\text { Cirurgia de } \\
\text { controle de } \\
\text { danos: } \\
\text { evolução nos } \\
\text { últimos } 20 \text { anos }\end{array}$ & $\begin{array}{l}\text { WAIBEL; } \\
\text { ROTONDO, } \\
\text { 2012, EUA }\end{array}$ & $\begin{array}{l}\text { A cirurgia para controle de danos tem } \\
\text { possibilitado a sobrevida de doentes } \\
\text { que sofreram lesões quase fatais } \\
\text { (lacerações visceral e vascular } \\
\text { concomitantes). }\end{array}$ \\
\hline $\begin{array}{l}\text { ARTIG } \\
09\end{array}$ & $\begin{array}{l}\text { Fatores de } \\
\text { risco para } \\
\text { mortalidade em } \\
\text { trauma } \\
\text { abdominal } \\
\text { contundente } \\
\text { com } \\
\text { abordagem } \\
\text { cirúrgica }\end{array}$ & $\begin{array}{l}\text { PIMENTEL et } \\
\text { al., 2015, } \\
\text { Brasil }\end{array}$ & $\begin{array}{l}\text { Os fatores de risco para óbito em } \\
\text { pacientes vítimas de trauma } \\
\text { abdominal fechado que necessitam de } \\
\text { laparotomia exploradora são: } \\
\text { instabilidade hemodinâmica como } \\
\text { indicação de laparotomia, rotura de } \\
\text { víscera maciça, múltiplas lesões intra- } \\
\text { abdominais, necessidade de controle } \\
\text { de danos cirúrgico, lesões graves }\end{array}$ \\
\hline
\end{tabular}


concomitantes e índice de trauma baixo.

Fonte: Autor próprio, 2020.

Foram incluídos 9 estudos em nossa revisão. Dentre os artigos analisados, um $(11,1 \%)$ discorreu sobre as formas de oclusão temporária da cavidade abdominal na laparotomia abreviada, um trabalho abordou a temática da cirurgia de controle de danos torácica $(11,1 \%)$ e $22,2 \%$ dos estudos investigaram as indicações e a importância da decisão desse tipo de abordagem. O impacto da cirurgia de controle de danos na redução da mortalidade dos doentes traumatizados foi abordado em $33,3 \%$ dos estudos incluídos. A cirurgia de controle de danos ortopédica, em casos de fratura, foi explanada em um estudo $(11,1 \%)$, assim como os fatores de risco para óbito no controle de danos abdominal, que foram abordados em um trabalho $(11,1 \%)$.

Estudo prospectivo de Pimentel et al. (2018), incluído em nossa revisão, foi realizado no Hospital do Trabalhador, centro de referência de trauma em Curitiba, Paraná, Brasil, e região metropolitana, e selecionou 46 pacientes internados entre janeiro de 2016 e fevereiro de 2017, vítimas de trauma penetrante ou fechado, que foram submetidos à cirurgia de controle de danos. Esse estudo analisou variáveis relevantes acerca dessa abordagem, como a idade, sexo e mecanismo de trauma mais prevalentes, indicações dessa abordagem, principais motivos e momento da decisão, além da sobrevida após cirurgia. Para isso, foi aplicado um questionário aos cirurgiões. Viu-se que a média de idade dos pacientes que precisaram de cirurgia para controle de danos foi de 34,2 anos $\pm 17,4$, e $97,8 \%(n=45)$ eram do sexo masculino. $O$ principal mecanismo de trauma foi o traumatismo penetrante, com ferimento por arma de fogo em 60,9\% ( $n=28)$ e ferimento por arma branca em 10,9\% $(n=5)$. A sobrevida foi de $80,4 \%(n=37)$, com nove óbitos. A maioria dos casos, $65 \%(n=30)$, foi atendida entre sexta-feira e domingo, sendo o horário mais prevalente entre $18 \mathrm{~h}$ e 23h59. Dois $(4,3 \%)$ cirurgiões tomaram a decisão de controlar o dano antes de o paciente chegar ao pronto socorro (PS), 26,1\% $(n=12)$ optaram assim que o paciente chegou no PS, 56,5\% ( $n=26)$ decidiram no início da cirurgia e 10,8\% $(n=5)$ decidiram pela piora do quadro durante a cirurgia. Em relação ao motivo que levou à decisão 
pela cirurgia de controle de danos, em 47,8\% $(n=22)$ dos casos foi a instabilidade hemodinâmica, com parada cardiorrespiratória em $2,2 \%$ dos casos $(n=1)$, e em $30,4 \%$ ( $n=14)$ foi a complexidade das lesões, sendo $8,7 \%(n=4)$ múltiplas lacerações de alças intestinais e 2,2\% ( $n=1)$ lesões cardíacas ou de grandes vasos, e em $8,7 \%$ ( $n$ =4), outros motivos. As alterações hemodinâmicas e laboratoriais levaram à escolha dessa abordagem, estavam presentes em $65,2 \%(n=30)$ dos pacientes, independente do momento de escolha. Onze (23,9\%) apresentavam alterações nos parâmetros hemodinâmicos, com choque grau III e IV, mas sem alterações laboratoriais, 4,3\% (n = 2) apresentavam apenas alterações nos exames laboratoriais e 6,5\% (n=3), não apresentavam alterações hemodinâmicas nem laboratoriais.

Análise retrospectiva de Kalil e Amaral (2016) foi realizada em um hospital público de referência na região metropolitana de Vitória, Espírito Santo, Brasil, e avaliou prontuários de 392 pacientes vítimas de trauma, selecionando 107 pacientes com lesões hepáticas por trauma, isoladas ou em associação a lesões de outros órgãos intra-abdominais, corrigidas à laparotomia exploradora, no período entre janeiro de 2011 a dezembro de 2013. Esse trabalho buscou estudar variáveis importantes acerca do trauma hepático e do controle de danos nesse contexto. Durante o tempo do estudo, 392 pacientes foram submetidos à laparotomia por trauma, 107 deles com lesão hepática com ou sem lesão intra-abdominal associada. Dos 107 pacientes, 93 eram homens $(86,9 \%)$ e $83,2 \%$ estavam nas primeiras quatro décadas de vida. $O$ mecanismo de trauma mais comum foi o trauma penetrante, em 84 pacientes $(78,5 \%)$, sendo 72 casos $(85,7 \%)$ por arma de fogo e 12 casos (14,3\%) por arma branca. Outras lesões intra-abdominais concomitantes ocorreram em 67 pacientes $(62,6 \%)$, sendo os órgãos mais acometidos o diafragma, cólon e estômago. Lesões extra-abdominais associadas foram encontradas em 77 pacientes (72\%), principalmente no tórax. A hepatorrafia foi a técnica cirúrgica mais utilizada $(80,37 \%)$ para o controle do sangramento hepático, e consequente controle dos distúrbios hemodinâmicos ligados ao choque hemorrágico. A cauterização da lesão hepática foi realizada em quatro pacientes $(3,74 \%)$ e em dois deles foi medida única e suficiente para interromper definitivamente a hemorragia. A ressecção de segmento hepático foi realizada em dois pacientes $(1,87 \%)$ e apenas um $(0,93 \%)$ necessitou de hepatectomia esquerda. $A$ 
cirurgia de controle de danos propriamente dita, com laparotomia abreviada e posterior reabordagem, foi aplicada em $6,5 \%$ dos pacientes, com sobrevida de $100 \%$ dos pacientes. Necessidade de transfusão de hemoderivados durante a cirurgia ocorreu em $26,2 \%$ dos casos, sendo utilizadas quantidades médias de 6,07 unidades de concentrado de hemácias e 3,01 unidades de plasma fresco congelado. Drenos foram colocados em 27 casos (25,2\%), sendo o dreno de Penrose o mais utilizado ( $\mathrm{n}$ = 15). A incidência de complicações pós-operatórias foi de $29,9 \%$, e as mais frequentes foram as infecciosas, incluindo pneumonia, peritonite e abscesso intraabdominal, que representaram $73,9 \%$ das complicações. A sobrevida geral dos pacientes vítimas de lesão hepática, associadas ou não à outras lesões, foi de $60 \%$ nos pacientes com trauma fechado e $87,5 \%$ nos pacientes com trauma penetrante. A mortalidade foi de $17,8 \%(n=19)$, sendo as causas de óbito mais comuns o choque hemorrágico, responsável por dez óbitos $(52,6 \%)$, e o choque séptico, com quatro óbitos (21\%).

Estudo de Waibel e Rotondo (2012), incluído em nossa revisão, demonstrou que em grandes séries, a cirurgia de controle de danos mostrou uma taxa de sobrevivência de aproximadamente $60 \%$, em comparação com a taxa de sobrevivência de $11 \%$ dos pacientes tratados convencionalmente e, em mecanismos de baixa energia, como ferimentos por arma branca, taxas até $90 \%$ foram relatadas. Esse estudo concluiu que a cirurgia para controle de danos tem possibilitado a sobrevida de doentes que sofreram lesões quase fatais (lesão vascular e visceral combinadas) e que, hoje, para sua aplicação original, a cirurgia de controle de danos alterou um evento quase uniformemente letal para aquele em que a maioria agora sobrevive.

Corroborando esses resultados de impacto do controle de danos na mortalidade dos pacientes graves, o estudo de Júnior, 2014, que reuniu informações de 25 artigos acerca do tema, observou que a laparotomia no controle de danos tem possibilitado maior sobrevida do que a esperada para o trauma abdominal. Diante desses resultados, sua aplicabilidade tem sido estendida para o trauma torácico e ortopédico. Nesse sentido, a revisão sistemática de Chavez et al. (2015), incluída em nosso estudo, investigou a aplicação das cirurgias de controle de danos no trauma 
ortopédico e demonstrou que a maioria dos artigos preconizou a estabilização da fratura nas primeiras 72 horas da lesão, no que concerne ao controle de danos, com essa abordagem estando associada à menor incidência de complicações, menor permanência hospitalar e na unidade de cuidados intensivos e à maior sobrevida.

\section{DISCUSSÃO}

\section{INDICAÇÕES DE CIRURGIA DE CONTROLE DE DANOS}

Em nosso estudo foi visto que a experiência clínica, até o presente momento, já demonstrou que quando a cirurgia para controle de danos é feita de maneira correta e, mais importante, quando devidamente indicada, eleva a sobrevida de pacientes vítimas de trauma. (EDELMUTH; BUSCARIOLLI; RIBEIRO JUNIOR, 2013). Sabe-se que a presença de sinais clínicos de instabilidade hemodinâmica, como taquicardia, hipotensão e taquipneia, alteração do nível de consciência, hipotermia e coagulopatia, são fatores indicativos da potencial necessidade de controle cirúrgico de danos. Além disso, estudos acerca das indicações mostram que o mecanismo do trauma e a gravidade das lesões, como lacerações vasculares, lesões de múltiplos órgãos, hemorragias graves em cavidades, também são fatores decisivos para esse tipo de abordagem.

Estudo prospectivo de Pimentel et al. (2018), incluído em nossa revisão, observou 46 pacientes internados em hospital de referência em trauma entre janeiro de $2016 \mathrm{e}$ fevereiro de 2017, vítimas de trauma penetrante ou contuso, submetidos à cirurgia para controlar danos. Os principais mecanismos de trauma foram traumatismo penetrante, com ferimento por arma de fogo em 60,9\% $(n=28)$ e ferimento por arma branca em 10,9\% ( $n=5)$. A sobrevida foi de $80,4 \%(n=37)$, com nove óbitos. Os fatores indicativos que levaram à decisão pela cirurgia de controle de danos, foram instabilidade hemodinâmica em 47,8\% ( $n=22)$, parada cardiorrespiratória em 2,2\% $(n=1)$ e gravidade das lesões em $30,4 \%(n=14)$, sendo lesões múltiplas de alças intestinais em $8,7 \%$ dos casos $(n=4)$, lacerações cardíacas ou vasculares em 2,2\% $(n=1)$, e em $8,7 \%(n=4)$ outros motivos. As alterações hemodinâmicas e laboratoriais que levaram à escolha dessa abordagem estavam presentes em $65,2 \%(n=30)$ dos 
pacientes, independente do momento de escolha. Em onze $(23,9 \%)$ havia alterações dos parâmetros hemodinâmicos condizentes com choque graus III e IV. Quanto ao tempo da indicação, na maioria dos casos, houve indicação precoce para cirurgia de controle de danos, logo que o paciente chegou ao PS, baseada principalmente no estado hemodinâmico e na gravidade das lesões, e em 65,2\%, a decisão foi compatível com alterações em dados hemodinâmicos e laboratoriais objetivos.

Corroborando esses resultados, análise retrospectiva de Asensio et al. (2001) avaliou, durante de 6 anos, 548 pacientes submetidos à cirurgia para controle de danos. Em relação ao mecanismo de trauma, as lesões penetrantes foram responsáveis por $82 \%$ das indicações versus lesões contusas 18\%. Acerca das alterações hemodinâmicas e laboratoriais, a pressão arterial média de $63 \mathrm{~mm} \mathrm{Hg}$, freqüência cardíaca de 78 batimentos por minuto, $\mathrm{pH}$ 7,15 e temperatura 34,3 ○ C. Nesse mesmo estudo, os fatores preditivos para mortalidade foram $\mathrm{pH} 7,2$, temperatura $<34{ }^{\circ} \mathrm{C}$, ou reposição de sangue $>4.000 \mathrm{~mL}$, reposição total de fluidos ou> $10.000 \mathrm{~mL}$, perda sanguínea estimada> $15 \mathrm{~mL} /$ minuto $(<0,001)$. Assim, o choque hipovolêmico, a hipotermia e a acidose, são as alterações hemodinâmicas e laboratoriais principais para a indicação dessa abordagem, uma vez que são potenciais causas de óbito em pouco tempo, caso não sejam revertidas, aventando, assim, a importância da indicação precoce e oportuna da cirurgia para controlar os danos. Além disso, o mecanismo de trauma e a gravidade das lesões também são importantes fatores indicativos, pois, a partir deles, pode-se prever a deterioração do quadro clínico do paciente para a tríade mortal do trauma, podendo o óbito ser inevitável. Em outro estudo incluído em nossa revisão, Pimentel et al. (2015) mostrou que a necessidade de cirurgia de controle de danos no trauma abdominal fechado é um próprio fator de risco para óbito, pois, na maioria dos traumas abdominais, os pacientes apresentam esses fatores de gravidade precocemente. 


\section{PAPEL DA CIRURGIA DE CONTROLE DE DANOS NA FISIOPATOLOGIA DO TRAUMA}

Os artigos 5, 7 e 8, indicados na Tabela I, incluídos em nossa revisão, apresentaram evidências de que a cirurgia de controle de danos eleva substancialmente a sobrevida de pacientes vítimas de trauma. Ao entendermos a fisiopatologia do trauma podemos compreender como as cirurgias de controle de danos atuam sobre ela, de maneira a reduzir o potencial de mortalidade das vítimas. Segundo Hameed; Aird e Cohn (2003), a hemorragia é principal complicação que leva à óbito o paciente politraumatizado. Coagulopatia secundária e lesão traumática grave se associam a pior prognóstico e morte. (HAMEED; AIRD; COHN, 2003.)

A hemorragia secundária ao trauma leva à hipoperfusão tecidual e choque hemorrágico. Além disso, o próprio trauma induz uma resposta imunometabólica, inflamatória e hemodinâmica que leva à exaustão das reservas fisiológicas, caso não haja reparo das lesões em tempo oportuno. Os mecanismos fisiopatológicos do trauma produzem graves anormalidades metabólicas secundárias à hemorragia, sendo as mais notáveis a coincidência de acidose, hipotermia e coagulopatia, a chamada tríade mortal do trauma. (SHAPIRO et al., 2000.). Segundo Burch et al. (1992), essa tríade, presente em pacientes gravemente feridos, é um ciclo vicioso que, se

ininterrupto, é rapidamente fatal.

O trauma é categorizado de acordo com os órgãos envolvidos. O politraumatizado é o indivíduo que apresenta duas ou mais lesões graves, sejam periféricas ou viscerais, que repercutem sobre a função respiratória ou circulatória e são fatais. As lesões traumáticas estão entre as cinco principais causas de morbidade e mortalidade em todo o mundo, sendo as lesões do sistema nervoso central e hemorragias os principais contribuintes deletérios. (ASENSIO et al, 2003.)

Estudo observacional analítico de Gonzalez Balverde et al. (2013), incluiu 100 pacientes politraumatizados, com idades entre 26 e 56 anos ( 90 homens), admitidos em uma sala de emergência, e visou estabelecer se a tríade mortal do trauma em pacientes politraumatizados está associada à maior mortalidade e à gravidade da 
lesão (ISS). Viu-se que existe uma relação entre a presença da tríade fatal com a gravidade da lesão avaliada pelo índice de gravidade da lesão (ISS), uma vez que os pacientes que apresentaram a tríade fatal tiveram escores de ISS de 30 ou mais. A presença da tríade fatal foi associada a maior mortalidade, independentemente do tipo de trauma. Na análise por grupos, traumas mistos e cranioencefálicos são os mais frequentes, sendo o aumento da pressão intracraniana e as hemorragias as causas diretas de morte. A presença da tríade fatal foi associada a maior mortalidade, portanto a tríade fatal poderia ser um indicador da magnitude da lesão e da oportunidade e relevância das manobras de reanimação implementadas e das cirurgias para controle de danos.

Os pacientes vítimas de trauma podem facilmente desenvolver a tríade letal, uma vez que frequentemente cursam com hemorragias graves, além de que são expostos em ambientes com baixas temperaturas, como salas de pronto-atendimento e de reanimação, e são submetidos à ressuscitação volêmica com soluções não aquecidas e, até mesmo, podem sofrer perda da capacidade de termorregulação, como em casos de intoxicação ou danos neurológicos. Esses fatores acarretam vasoconstrição e consequente hipoperfusão periférica, que têm início a partir de uma descarga adrenérgica. A hipóxia resultante ocasiona a conversão do metabolismo aeróbico em anaeróbico, que culmina em acidose metabólica. A hipotermia, por sua vez, contribui de diversas maneiras para o desenvolvimento de coagulopatia, pois inibe a interação entre fatores de coagulação e plaquetas, ocasiona disfunção endotelial e do sistema fibrinolítico e inativa fatores temperatura-dependentes, os quais, em temperaturas menores que $35^{\circ} \mathrm{C}$, entram em um estado de hipometabolismo e hipofunção, agravando hemorragias e deteriorando o quadro clínico do paciente.

Burch et al. (1992) discorreram acerca desse ciclo vicioso de descontrole metabólico e exaustão fisiológica. Hemorragia resultante de distúrbio de coagulação apresenta taxa de $40 \%$ quando associada à hipotensão, $50 \%$ quando presente à hipotermia e $60 \%$ na presença de acidose, podendo atingir $98 \%$ de ocorrência quando do desenvolvimento de toda a tríade letal. Dessa forma, a hemorragia nos pacientes politraumatizados, por lesões vasculares, viscerais, fraturas de ossos longos, na 
grande maioria dos casos, é a causa base que desencadeia esse ciclo, e, se não interrompida oportunamente, pode ser fatal. Nesse ponto, entra a abordagem cirúrgica para o controle dos danos, que visa reparar a fonte do sangramento, cessando a hemorragia para, assim, impedir o desencadeamento da da tríade mortal.

\section{CONTROLE DE DANOS ABDOMINAL: CONTROLE DA HEMORRAGIA E DA CONTAMINAÇÃO}

No contexto do controle de danos abdominal, estudo de Kalil e Amaral (2016), incluído em nossa análise, apresentou resultados de sobrevida geral dos pacientes vítimas de lesão hepática, associadas ou não à outras lesões e submetidos à laparotomia exploradora, de $60 \%$ nos pacientes com trauma fechado e $87,5 \%$ nos pacientes com trauma penetrante. A mortalidade foi de $17,8 \%(n=19)$, sendo as causas de óbito mais comuns o choque hemorrágico, responsável por dez óbitos $(52,6 \%)$, e o choque séptico, com quatro óbitos (21\%). (KALIL; AMARAL, 2016).

Diante disso, além da interrupção da hemorragia, principal causa base de óbito no contexto do trauma abdominal, o controle da contaminação da cavidade peritoneal também faz parte das estratégias de controle de danos abdominal. A contaminação cavitária pode ocasionar sepse e choque séptico rapidamente e, quando associada à hemorragia, leva à exaustão fisiológica e à morte. Diante disso, rafias do trato gastrointestinal são feitas nas cirurgias de controle de danos abdominal, com fechamento temporário da cavidade, colostomias temporárias, drenos e diferentes estratégias para o reparo de lesões potencialmente fatais e controle da contaminação. Na reabordagem em segundo tempo cirúrgico, o reparo definitivo e o fechamento da cavidade são realizados. O estudo de Burch et al. (1992) observou 200 pacientes, durante 7,5 anos, que foram tratados com laparotomia ou toracotomia abreviadas, com o objetivo de interromper o ciclo fatal. Cento e setenta pacientes (85\%) sofreram lesões penetrantes e 30 (15\%) foram vítimas de trauma contuso. Toracotomia reanimadora foi realizada em 60 (30\%) pacientes. As técnicas para abreviar a laparotomia incluíram a ligadura de lesões entéricas em 34 pacientes, pinças vasculares retidas em 13, shunts intravasculares temporários em quatro, 
tamponamento de superfícies com sangramento difuso em 171 e o uso de vários clipes de toalha para fechar apenas a pele da parede abdominal em 178. Os pacientes foram então transportados para a unidade de terapia intensiva cirúrgica para correção vigorosa de distúrbios metabólicos e coagulopatias. Noventa e oito pacientes (49\%) sobreviveram para se submeterem à reoperação planejada (atraso médio de 48,1 horas), e 66 de 98 (67\%) sobreviveram para deixar o hospital.

\section{FECHAMENTO TEMPORÁRIO DA CAVIDADE ABDOMINAL NO CON TEXTO DO CONTROLE DE DANOS}

$\mathrm{Na}$ laparostomia para controle de danos, os planos da parede abdominal não são fechados por completo, permitindo uma inspeção regular do conteúdo intracavitário e reoperações. O estudo de Ribeiro Jr et al. (2016), incluído em nossa revisão, comparou diferentes técnicas de fechamento temporário do abdome em laparostomias de controle de danos. Viu-se que a técnica VAC foi superior em relação às técnicas de bolsa Bogotá e de Barker, por apresentar benefícios como menor ocorrência de fístulas, infecções e outras complicações, menor morbimortalidade, melhor controle do líquido do terceiro espaço e maior facilidade no fechamento primário da parede abdominal. Com eficácia em feridas traumáticas graves, a técnica VAC era utilizada principalmente em cirurgia plástica e reconstrutiva e vem sendo estendida às laparostomias temporárias de controle de danos, pelas vantagens que apresenta. Estudo experimental de Wang et al. (2014) investigou o efeito da VAC na liberação de citocinas inflamatórias, e mostrou, em seus resultados, que o sistema VAC proporciona aumento local de neutrófilos, interleucinas e fatores de crescimento endotelial vascular, estímulo à angiogênese, remoção de fluidos do terceiro espaço e resíduos, otimização do suprimento de sangue e deposição de matriz, além de diminuição da proliferação bacteriana e aumento da pressão parcial de oxigênio nos tecidos, exercendo, assim, mecanismo multimodal na prevenção de complicações, em consonância com os resultados de nossa revisão. Dessa maneira, a manutenção da laparostomia para regulares inspeções da cavidade abdominal e posteriores reabordagens é otimizada quando utilizada a técnica VAC. 


\section{CIRURGIA DE CONTROLE DE DANOS TORÁCICA}

O controle de danos no trauma torácico geralmente é iniciado com medidas simples, como drenagem torácica e oxigenoterapia, muitas vezes suficientes para a estabilização do doente. Porém, alguns pacientes irão necessitar de intervenção cirúrgica por meio de toracotomia, devido hemotórax maciço com choque hipovolêmico, lesão vascular ou esofágica, lacerações cardíacas, lesões pulmonares graves prejudicando a ventilação, condições que requerem reparo imediato, a fim de restaurar a normalidade fisiológica do paciente traumatizado. A toracotomia de emergência configura-se como a abordagem mais desafiadora dentro do contexto do controle de danos em cirurgia, pois requer o controle agressivo, rápido e, muitas vezes, parcial ou temporário de lesões com alto potencial de mortalidade. Segundo Gonçalves e Saad (2016), os principais objetivos de uma toracotomia de emergência são a resolução do tamponamento cardíaco, através da pericardiocentese, reparo de lacerações cardíacas ou feridas exsanguinantes devido lesão vascular, pinçamento da aorta descendente para aumentar o fluxo sanguíneo coronário e cerebral, compressões cardíacas internas e oclusão do hilo pulmonar para reduzir o risco de embolia gasosa e diminuição do sangramento em lesões pulmonares graves.

Para alcançar esses objetivos primordiais, o intuito do controle de danos torácico é realizar o reparo menos definitivo, utilizando as técnicas mais rápidas e fáceis para diminuir o tempo operatório. Algumas lesões podem ser parcialmente reparadas e requererão reoperação logo que o paciente apresente melhora e estabilidade clínica e hemodinâmica. (ROTONDO; BARD, 2004). Estudo de Gonçalves e Saad (2016), incluído em nossa revisão, mostrou que manobras relativamente simples podem abreviar o tempo operatório de pacientes graves no contexto do controle de danos torácico e diminuir consideravelmente a mortalidade desses pacientes. Em semelhança a esses achados, estudo de Mattox e Walkes (2006) concluiu que, no trauma torácico, sangramento ativo externalizado através de uma ferida aberta, como em ferimentos penetrantes, ou sangramento ativo ou contido em compartimento mediastinal, pleural ou extrapleural, o controle imediato e temporário do sangramento 
pode ser feito com a introdução de cateter de Foley na ferida, insuflação do balão e tração do cateter. Se a ferida for extensa na pele, pode-se aplicar pontos ao redor do cateter e fixá-lo. Essas são medidas simples que podem evitar o desenvolvimento de choque hipovolêmico e a evolução para a tríade fatal. Segundo Rotondo e Bard (2004), nas lacerações ventriculares, o sangramento também pode ser controlado, inicialmente, por oclusão digital ou introdução de cateter de Foley através da lesão e insuflação do balão, após isso, sutura ventricular (cardiorrafia) com fio inabsorvível e agulha do tipo vascular em pontos em "U" ou "Halsted" deve ser realizada sempre que possível, como controle temporário e eficiente do sangramento e disfunção cardiovascular, medidas relativamente simples que salvam a vida do paciente vítima de trauma cardíaco.

\section{CONCLUSÃO}

Diante dos dados coletados da literatura e explanados neste estudo, conclui-se que a cirurgia de controle de danos vem sendo uma modalidade efetiva em reduzir a mortalidade de pacientes graves e tem se expandido para além das lesões abdominais, com a laparotomia abreviada, sendo também aplicada aos casos de trauma torácico, fraturas e, até, em condições não-traumáticas que exigem controle imediato de sangramento e/ou contaminação e resgate da fisiologia do doente. Com isso, a partir desses principais achados da presente revisão, salienta-se a importância de os cirurgiões conhecerem as implicações teóricas e práticas dessa abordagem, suas indicações, o modo como atua na fisiopatologia do trauma, interrompendo a tríade letal e preservando as reservas fisiológicas, o momento da decisão, tempo até a reabordagem, etapas do controle de danos, além de suas diversas aplicabilidades e técnicas. O conhecimento acerca dessa modalidade permitirá ao cirurgião reconhecer prontamente o paciente que necessita ser submetido ao controle de danos e decidir pela cirurgia no momento oportuno, além de realizar técnica correta, muitas vezes simples, mas de extrema efetividade, e planejar a reabordagem, assim como a reabilitação. Dessa forma, a mortalidade dos pacientes graves, portadores da tríade letal e de esgotamento das reservas fisiológicas, reduzirá ainda mais, uma vez que a 
cirurgia de controle de danos, quando bem indicada e realizada, eleva sobremaneira a sobrevida desses pacientes.

\section{REFERÊNCIAS}

ASENSIO, Juan A. et al. Gerenciando a exsanguinação: o que sabemos sobre controle de danos / resgate não é suficiente. In: Procedimentos do Baylor University Medical Center. Taylor e Francis, 2003. p. 294-296. 2003.

ASENSIO, Juan A. et al. Variáveis confiáveis no paciente exsanguíneo que indicam controle de danos e predizem o resultado. The American Journal of surgery, v. 182, n. 6, pág. 743-751, 2001.

$\mathrm{BURCH}, \mathrm{J}$. M. et al. Abbreviated laparotomy and planned reoperation for critically injured patients. Annals of surgery, v. 215, n. 5, p. 476, 1992.

CHAVEZ, Javier Peña et al. Controle de danos em fraturas instáveis torácicas e lombares em politrauma. Revisão sistemática. Coluna / Columna, São Paulo, v. 14, n. 2, pág. 153-156, junho de 2015.

EDELMUTH, Rodrigo Camargo Leão; BUSCARIOLLI, Yuri dos Santos; RIBEIRO JUNIOR, Marcelo Augusto Fontenelle. Cirurgia para controle de danos: estado atual. Rev. Col. Bras. Cir., Rio de Janeiro, v. 40, n. 2, p. 142-151, Apr. 2013.

GERMANOS, S.; GOURGIOTIS, S.; VILLIAS, C. et al. Cirurgia de controle de danos no abdômen: uma abordagem para o tratamento de pacientes com lesões graves. Int J Surg. V. 6(3): p. 246-52. 2008.

GONCALVES, Roberto; SAAD JR, Roberto. Cirurgia de controle de danos torácicos. Rev. Col. Bras. Cir., Rio de Janeiro, v. 43, n. 5, pág. 374-381, outubro de 2016. 
GONZALEZ BALVERDE, María et al. Valor prognóstico da tríade letal em pacientes politraumatizados. Rev. méd. Chile, Santiago, v. 141, n. 11, pág. 14201426, novembro de 2013.

HAMEED, SM; AIRD, WC e COHN, SM. Entrega de oxigênio. Medicina intensiva, v. 31, n. 12, pág. S658-S667, 2003.

JÚNIOR, Aloísio Cardoso. Controle de danos: uma luz no fim do túnel. Rev. méd. Minas Gerais, v. 24, n. 4, 2014.

KALIL, Mitre; AMARAL, Isaac Massaud Amim. Avaliação epidemiológica de vítimas de trauma hepático submetidas à cirurgia. Rev. Col. Bras. Cir., Rio de Janeiro, v. 43, n. 1, pág. 22 a 27 de fevereiro de 2016.

MATTOX, K.L. E WALKES, J.C. Avanços no tratamento da lesão vascular torácica. Scan J Surg. Vol. 91 (1): págs 46-51. 2006.

PIMENTEL, SILVÂNIA KLUG et al. Cirurgia de controle de danos: estamos perdendo o controle das indicações? Rev. Col. Bras. Cir. , Rio de Janeiro, v. 45, n. 1, e1474, 2018.

PIMENTEL, SILVANIA KLUG et al. Fatores de risco para mortalidade em trauma abdominal fechado com abordagem cirúrgica. Rev. Col. Bras. Cir. , Rio de Janeiro, v. 42, n. 4, pág. 259-264, agosto de 2015.

RIBEIRO JR, MARCELO A. F. et al. Estudo comparativo de técnicas de fechamento temporário da cavidade abdominal durante o controle de danos. Rev. Col. Bras. Cir. Rio de Janeiro, v. 43, n. 5, p. 368-373, out. 2016.

ROTONDO M.F. e BARD M.R. Damage control surgery for thoracic injuries. Injury. Vol. 35, ed.7, págs 649-654, 2004.

ROTONDO, Michael MF; SCHWAB, CW; MCGONIGAL MD, et al. Controle de danos: uma abordagem para melhorar a sobrevida em lesão abdominal penetrante exsanguinante. Jornal Trauma. V. 35(3): p. 375-82; discussão 382-3. 1993. 
SHAPIRO, Michael B. et al. Controle de danos: revisão coletiva. Journal of Trauma and Acute Care Surgery, v. 49, n. 5, pág. 969-978, 2000.

SIMAO, Tiago Sarmento et al . Curativo à vácuo para cobertura temporária de peritoneostomia. ABCD, arq. bras. cir. dig., São Paulo , v. 26, n. 2, p. 147150, June 2013.

WAIBEL, Brett H; ROTONDO, Michael MF. Cirurgia de controle de danos: sua evolução nos últimos 20 anos. Rev. Col. Bras. Cir., Rio de Janeiro, v. 39, n. 4, pág. 314-321, agosto de 2012.

WANG, Weiyang et al. "O fechamento assistido por vácuo aumenta a expressão de ICAM-1, MIF, VEGF e colágeno I na terapia de feridas". Medicina experimental e terapêutica. vol. 7,5 (2014): 1221-1226. 2014.

Enviado: Dezembro, 2020.

Aprovado: Janeiro, 2021. 